\title{
The two faces of p73
}

\author{
TJ Grob ${ }^{1}$, MF Fey ${ }^{1}$ and A Tobler ${ }^{\star, 1}$ \\ ${ }^{1}$ Department of Clinical Research and Medical Oncology/Hematology, \\ University and Inselspital, Bern, Switzerland \\ * Corresponding author: A Tobler, Department of Clinical Research and Medical \\ Oncology/Hematology, University and Inselspital, Bern, Switzerland, \\ Tel: +41316329601 or +41 31632 8816; E-mail: andreas.tobler@insel.ch \\ DOI: $10.1038 / \mathrm{sj} / \mathrm{cdd} / 4401018$
}

In the present issue of Cell Death and Differentiation, Casciano et al., report that in neuroblastoma patients expression of the anti-apoptotic $\Delta N$ variant of p73 is strongly associated with reduced survival and predicts a poorer outcome independent of age, primary tumor site, stage and MYCN amplification. ${ }^{1}$ Yet, the very same gene is also able to cause apoptosis and inhibition of cell proliferation $^{2}$ in cancer cells. How do we resolve this apparent paradox of a gene gazing in two opposite directions?

In 1997, when p73 was described as the first p53 homologue, ${ }^{3}$ the obvious question was whether p73 would be the long sought after tumor suppressor at $1 p 36$, a locus which is commonly deleted in various tumor entities. In fact, p73 is located at 1p36, and shows tumor suppressor functions. ${ }^{3}$ This finding initiated an extensive search of the p73 status in primary tumors, particularly those with 1 p36 deletions, as is the case with neuroblastomas. ${ }^{4}$ Stiewe and Pützer review the results of this frustrating endeavour. ${ }^{5}$ More than 1000 tumors were screened, and p73 mutations were quite rare: less than $0.5 \%$, compared to circa $50 \%$ found in p53. This is also true for neuroblastoma, where only two mutations (P405R, P425L) in the p73 gene have been found in 302 tumors. $^{5}$ Intriguingly, neuroblastoma does not show significant mutations in p53 either. ${ }^{4}$ Still, if we take as an example the case of neuroblastoma, the presence of significant $\mathrm{LOH}$ implies the involvement of p73 in the development of the cancer. ${ }^{5}$ This implies that p73 is not a classical tumor suppressor gene and, if it does play a role in cancer, it would not follow Knudson's two hit hypothesis.

Thus, the p73 story is more complex than it would be for classical tumor suppressor genes, such as its sibling p53. In fact, the intricacies of p73 regulation are only now beginning to be understood. p73 is expressed as distinct forms differing at either at the $\mathrm{C}$ - or the $\mathrm{N}$-terminus. Differential splicing of the $3^{\prime}$ end leads to the expression of several p73 C-terminal splice variants that are able to homo- and -heterodimerize, and that vary in their potential to activate p53-responsive genes, such as p21 Waf1/Cip1 and Bax. $^{6}$ More importantly, a p73 variant lacking the $\mathrm{N}$ - terminal transactivation domain $(\Delta \mathrm{Np} 73)$ was described in mice. ${ }^{7,8}$ This complex patter of isoforms and their related functions has been outlined in several recent reviews. ${ }^{9,10}$ The human $\Delta \mathrm{Np} 73$ was only described in December 2001. ${ }^{11}$ Currently, six different C-terminus splicing variants have been found in normal cells; and the $\Delta \mathrm{Np} 73$ form seems to be produced in all possible C-terminus types, creating an increasing degree of complexity. The human $\Delta \mathrm{N}$ variant is independently regulated by its own promoter, located in intron 3, and inhibits the transactivating p73 variant (TAp73) in a dominant negative fashion. The $p 53-\Delta N p 73$ relationship is extremely close: $\Delta N p 73$ regulates p53 (as well as TAp73) function since it is able to block its transactivation activity and its ability to induce apoptosis. This inhibitory function can be exerted either at oligomerization level (as for TAp73), or by competing for the same target sequence (as for p53). ${ }^{11}$ On the other hand, the $\Delta \mathrm{Np} 73$ promoter contains a very efficient p53/ p73 responsive element. Consequently, $\Delta \mathrm{Np} 73$ is also induced by p53 creating a dominant negative feedback loop. ${ }^{11}$

Hence the TP73 locus encodes both a tumor suppressor (TAp73) and a putative oncogene $(\Delta \mathrm{Np} 73)$, raising important questions on its possible role in tumorigenesis. The facts and the problems are the following. (i) Increased expression of p73 was found in tumor tissues compared with their normal counterparts (reviewed by Stiewe and Pützer). ${ }^{5}$ However, these studies do not answer the question which of the two p73 variants is overexpressed and ultimately plays a role in tumorigenesis. (ii) p73 knock out mice lack both the apoptotic (TAp73) and the antiapoptotic $(\Delta \mathrm{Np} 73)$ variants. $^{7}$ This might well be the reason why they do not develop spontaneous tumors. Knock out mice with specific deletions of the respective variants should help to tackle this unresolved paradox. (iii) Since the same exons of p73 encode the DNA-binding domains of both TAp73 and $\Delta \mathrm{Np} 73$, respective mutations (which are frequent in p53) would inactivate the p73 tumor suppressor variant and suppress $\Delta \mathrm{Np} 73$ oncogenic activity. As a corollary, no mutations were found in the open reading frame of TAp73.

Since Knudson's two hit hypothesis is not applicable to p73, two possibilities remain. Either, 1p36 harbours a tumor suppressor gene other than p73. Or, if p73 is hit by $\mathrm{LOH}$ at $1 \mathrm{p} 36$, one would imagine a more complex model system than that of Knudson. One could anticipate, for example, the loss of one allele at 1p36 and mutations in the promoter region of the apoptotic TAp73. A first step towards an answer to this puzzling and unresolved issue, is to distinguish between the expression of the two variants in tumor tissue. Casciano et al., show that in neuroblastoma patients expression of $\Delta \mathrm{Np} 73$ and not of 
TAp73 correlates with both poor overall and progression free survival. ${ }^{1}$ Overexpression of $\Delta \mathrm{Np} 73$ could thus generate a functional block of p53. The net result on a cancer cell would mirror the effect of the structural p53 inactivation, and a functionally p53 negative, aggressive tumor would results. How are these two crucial promoters, coding for TAp73 or $\triangle \mathrm{Np} 73$, regulated? $\mathrm{A}$ preliminary attempt at understanding this question is given in the companion Letter by the same Authors, suggesting a selective control by methylation in the $\Delta$ Np73 promoter. ${ }^{12}$

Since its discovery as a younger sibling of p53, p73 has always been good for a surprise. As it now stands, p73 is a gene with two faces looking in opposite directions. Which one of the two faces is more important for cell behavior and cancer biology has yet to be elucidated.

1. Casciano I et al. (2002) Cell Death Differ. 9:246-251

2. Jost CA et al. (1997) Nature 389: 191-194

3. Kaghad M et al. (1997) Cell 90: 809-819

4. Broder GM et al. (1997) J. Pediatr. Hematol. Oncol. 19: 93-101

5. Stiewe T and Pützer BM. (2002) Cell Death Differ. 9: 237-245

6. De Laurenzi V et al. (1998) J. Experim. Med. 188: 1763-1768

7. Yang A et al. (2000) Nature 404: $99-103$

8. Pozniak CD et al. (2000) Science 289: 304-306

9. Yang A and McKeon F (2000) Nat. Rev. Mol. Cell. Biol. 1: 199-207

10 Levrero M, De Laurenzi V (2000) J. Cell Sci. 113(10): 1661-1670

11 Grob TJ et al. (2001) Cell Death Differ. 8 (12): 1213-1223

12 Casciano I et al. (2002) Cell Death Differ. 9: 343-345 